\title{
Psicología Básica y Psicología Aplicada
}

\author{
Soledad Ballesteros Jiménez
}

\section{Introducción}

Hoy vamos a hablar de Psicología Básica y Psicología Aplicada, y lo vamos desde la perspectiva de la Psicología como ciencia cuyo objcto de estudio es la conducta y la mente de los organismos, y especialmente del hombre.

La Psicología el método científico para obtener el conocimiento. Esto significa que los psicólogos nos adherimos a ciertas reglas que exigen la medición pública y objetiva de la conducta, la puesta a prueba de hipótesis y su contrastación experimental. Debido a la importancia que el paradigma experimental ha tenido y tiene hoy para la Psicología, nos detendremos a comentar una seric de criterios en los que se basa este paradigma, para pasar a continuación a revisar cuáles han sido los logros de la investigación básica en Psicología teniendo en cuenta cuáles han sido y siguen siendo los premios 0 reforzadores más importantes que se utilizan en este terreno. Haremos especial hincapié en uno de ellos, el de la publicación, y los problemas que plantean los criterios actuales de publicación de trabajos de investigación.

A continuación nos detendremos en la investigación aplicada y señalaremos las dos posturas que existen actualmente en torno a este tema:

- La que defiende que la investigación aplicada no es sino la aplicación de los principios teóricos derivados de la investigación básica a la solución de los problemas importantes que se plantean.

- La que defiende que investigación básica e investigación aplicada funcionan como dos paradigmas diferentes, el de la ciencia y el de la tecnología.

Finalizaremos nuestra intervención proponiendo un posible plan de acción para la Psicología tanto a nivel de la investigación básica como a nivel de la investigación aplicada.

\section{Investigación Básica e Investigación Aplicada}

La edición de 1850 del Webster Dicconary definia la Psicología como "Discurso o tratado del alma humana, o la doctrina de la naturaleza espiritual del hombre" ( $\mathrm{p}$. 886). El Webster's New Collegiate Diccionary en su edición de 1985 la defie como "Ciencia de la mente y de la conducta (p. 951). El cambio experimentado en la definición es lo suficientemente importante como para que nos detengamos un momento en cuáles han sido lo principales acotecimientos que han influido en este cambio desde el discurso filosófico al estudio científico. 
Fueron ciertos acontecimientos ocurridos en torno al laboratorio de Wilhelm Wund en Leipzig (Alemania), los responsables de que la Psicología se convirtiera en una ciencia. Wund, después de formarse como fisiólogo en heidelberg y Tübingen, de haber trabajado en Berlin también como fisiólogo, ocupa la cátedra de filosofía en Zurich, para pasar más tarde a Leipzig donde comienza a enseñar psicología. Fue en este ciudad donde fundó el primer laboratorio de Psicología en 1879. En este laboratorio se formaron una serie de psicólogos europeos y americanos que más tarde extendieron los presupuestos de la nueva ciencia por Europa y Estados Unidos. Entre los temas estudiados por Wund y sus discípulos destacan la sensación y la percepción que son los dos temas principales. Otros temas fueron el tiempo de reacción, la cronometría mental y la atención.

En aquel momento el objeto de la nueva ciencia era la conciencia y su método de estudio la introspección. Para Wundt la Psicología es una ciencia experimental y la introspección debe realizarse bajo condiciones controladas experimentalmente.

A finales del siglo pasado, Estados Unidos empezó a enviar jóvenes estudiantes al laboratorio de Wundt para que se formaran en los métodos de la nueva ciencia. Uno de esos estudiantes fue J. Mackeen Cattell que se doctrinó en Alcmania en la Universidad de Leipzig hace poco menos de un siglo, en 1896, y fue el primer norteamericano que terminó su tesis bajo la dirección de Wilhem Wundt. Este joven regresó a Estados Unidos y fundó los laboratorios de Psicología de las Universidades de Pensilvania y Columbia, siendo considerado como el gran impulsor de la Psicología Diferencial en Norteamérica. Pero lo más imporante es que Cattell creía en el valor de la nueva ciencia. El mismo escribía en una carta dirigida a sus padres:

"La Psicología parece que va a ser la ciencia de los próximos 30 años, y claramente, la ciencia en la que se van a realizar los mayores progresos".

Por aquella época, las universidades americanas buscaban profesores capaces de enseñar la nueva ciencia marcando de esta manera los comienzos de la Psicología Experimental. La Psicología tuvo tanto éxito en el marco académico que en 1903, 20 años después de que Stanley Hall, pionero junto con William James de la Psicología Experimental en su país, fundara el primer laboratorio de psicología en Estados Unidos en Johns Hopkins University ya existían 50 laboratorios que habian producido 100 doctores en esta nueva ciencia. Sin embargo, a pesar de este rápido desarrollo, poca gente fuera de los ámbitos académicos parecía comprender lo que era la Psicología. La gente equiparaba Psicología con clarividencia, lectura de la mente y espiritualismo.

El acontecimiento que empezó a cambiar esta situación fue precisamente el movimiento de la Psicología hacia los campos aplicados, especialmente el de la Educación. En unos momento en los que Estados Unidos necesitaba reformar su sistema educativo para atender al gran número de emigrantes y al rápido crecimiento de las ciudades, tuvieron una gran influencia algunos norteamericanos que estudiaron a finales de siglo en Alemania y que siguieron las teorias de Herbart. Este autor defendía que la Educación debía basarse en la Psicología. Así pues, la Educación parecía un buen campo para que la Psicología aplicara sus nuevos conocimientos.

Stanley Hall escribió en cierta ocasión:

"El principal e inmediato campo de aplicación de la Psicología es su aplicación a la Educación". 
Se pensaba que con los nuevos métodos experimentales la Psicología sería capaz de conocer todo acerca del nifno, sus capacidades sensoriales, características físicas, memoria, juego, atención, etc. Haciendo uso de estos nuevos conocimientos la Pedagogía no sería más una adivinación sino una ciencia. Las técnicas educativas podrían planificarse de mancra que resultaran efectivas para todo tipo de estudiantes.

Stanley Hall fue uno de los psicólogos más destacados de su tiempo que promovió y defendió activamente el valor de la Psicología Aplicada. Desde sus comienzos en el área de la Educación, la Psicología Aplicada continuó su crecimiento extendiéndose a otras áreas tales como la práctica clínica, los negocios o la industria. Muchas de estas aplicaciones prácticas se centraron en aquel momento en el uso de los tests psicológicos.

En torno a los años 20, el público americano parecía creer en la nueva ciencia de la Psicología ya que veía en ella una ayuda importante para conseguir el éxito y la prosperidad. La demanda de los servicios psicológicos fue muy grande, mayor de la que podían atender el número de psicólogos existentes en aquel momento. Esta demanda hizo que muchos desaprensivos, con poca o ninguna preparación en Psicología empezaran a ofrecer sus servicios profesionales como Psicólogos. Esta situación, llegó a preocupar al APA (la mayor asociación de Psicólogos de Estados Unidos) que como ha scñalado Banjamin (1986), llegó a establecer un certificado para poder ejercer como psicólogos, pero tuvo problemas y terminó aboliéndose.

La popularidad de la Psicología aumentó todavía más después de la Primera Guerra Mundial debido a la importancia que alcanzaron los piscólogos que trabajaban en el ejército en tareas de selección, y al éxito de los tests colectivos que se utilizaron para estos menesteres. Al mismo tiempo, los psicólogos clínicos empezaron a trabajar en el ámbito aplicado debido sobre todo a sus experiencias después de la guerra con los veteranos de guerra que habían sufrido lesiones cerebrales o simplemente presentaban problemas psicológicos como consecuencia de su estancia en el frente.

Desde entonces, la Psicología Aplicada ha tenido momentos altos y bajos, pero lo que no puede dudarse es de su relevancia y valor para solucionar importantes problemas que el ser humano tiene planteados.

Partiendo de la definición que acabamos de dar de la Psicología como ciencia de la mente y de la conducta, parece claro que el punto central de toda actividad psicológica es la investigación básica y la investigación aplicada ya que es a través de la investigación como se desarrolla el conocimiento científico. Este conocimiento, se utiliza después para proporcionar servicios profesionales en el campo educativo, clínico y organizacional. Es precisamente por la vocación aplicada de la Psicología por lo que esta ciencia se ha mantenido a lo largo de sus más de 100 años de existencia como tal y ha experimentado el enorme crecimiento cuantitativo que se está registrando en los últimos años. Creemos que se puede afirmar que si la psicología hubiese sido únicamente una ciencia pura y los psicólogos no hubiesen considerado la necesidad de aplicar sus conocimientos y explotarlos de una manera responsable, la psicología hubiera permanecido siempre pequeña y posiblemente estéril. Fueron precisamente sus posibilidades de aplicación las que dieron a nuestra ciencia una enorme popularidad. La Psicología se convirtió en una profesión sumamente atractiva para muchos jóvenes tanto en Estados Unidos como en Europa y, naturalmente, en España. 
Como ejemplo de este crecimiento, observemos los siguientes gráficos proporcionados por Mckinney (1976) sobre el desarrollo cuantitativo de la Psicología Norteamericana hasta la década de los 70 . La Figura 1 muestra el crecimiento portentoso experimentado en todos los aspectos de la Psicología norteamericana en lo que va de siglo.

\section{CRECIMIENTO CUANTITATIVO DE LA PSICOLOGIA AMERICANA ( Tomado del American Psychologist) datos de Mckinney}

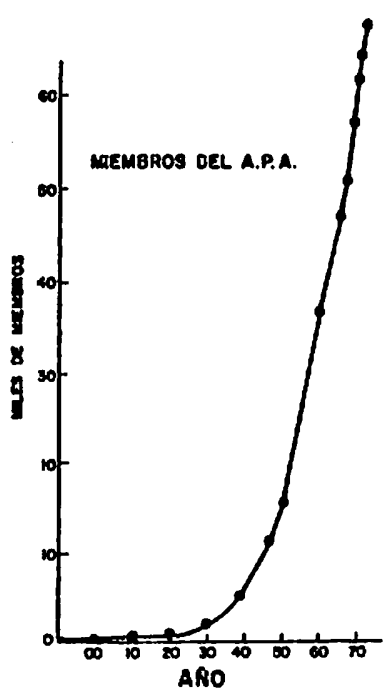

F1G. IA

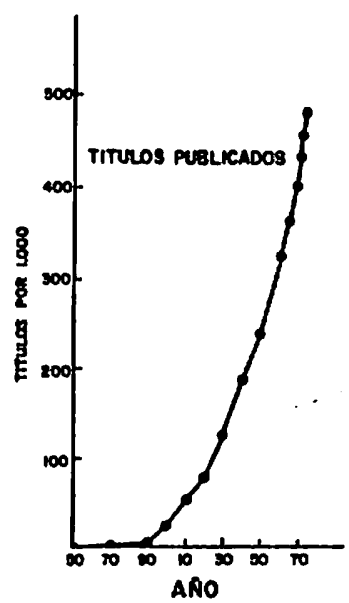

FI6. IA

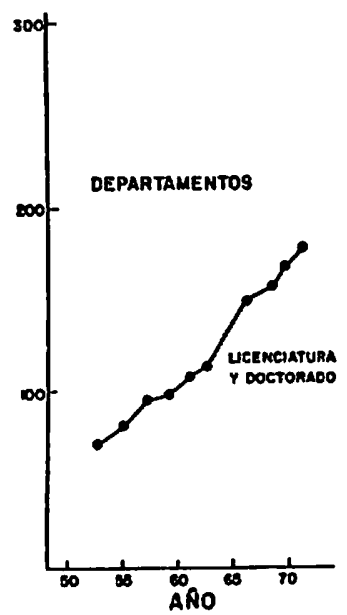

FIG. IC

Figura 1 
La Figura 1 A muestra el crecimiento experimentado por el número de afiliados al APA, American Psychological Association, la asociación más importante de psicólogos que existen en la actualidad.

La Figura $1 \mathrm{~B}$, muestra el aumento en el número de libros publicados desde 1850 a 1970 en millares.

La Figura $1 \mathrm{C}$, expresa el número de Departamentos que ofrecen estudios de licenciatura y doctorado en Psicología y el aumento experimentado entre los años 1950 a 1970.

Si ésto ha ocurrido en Estados Unidos, no menos cabe decir de lo ocurrido en nuestro país, aunque mucho después. Considerando que los estudios de Psicología como disciplina universitaria no comenzó hasta 1968, como una especialización dentro de la carrera de Filosofia y Letras, su desarrollo ha sido enorme. Hasta esa fecha, los únicos estudios de Psicología que existían en España eran los de las Escuelas de Psicología de Madrid y Barcelona. A estas escuelas podían acceder licenciados, después de finalizar cualquier carrera universitaria, y los estudios consistían en dos cursos académicos durante los cuales se estudiaban una serie de asignaturas según la especialización elegida: clínica, escolar o industrial.

El número de Diplomados de la Escuela de Psicología de Madrid durante los 16 años de funcionamiento de la Escuela hasta que salió la primera promoción de licenciados, fue de 1746. Según datos proporcionados por el Colegio de Psicólogos, actualmente existen en España más de 25.000 psicólogos que han salido de nuestras universidades desde 1971. Desde el momento que se instituyó la carrera de Psicología su aceptación por parte de los estudiantes fue muy grande. La mayor explosión se produjo durante el curso 1976-1977. Este curso, el número de alumnos matriculados en Psicología de la Complutense fue de 9.000 . A partir de esa fecha el número de alumnos ha descendido ligeramente o se ha mantenido, pareciendo que se ha tocado techo. Por lo que respecta a la UNED, nuestra carrera es la segunda más estudiada a continuación de la Derecho, y durante el curso $86 / 87$ la matricula ascendió a 8.900 alumnos.

En la actualidad, como ha señalado recientemente Richard Snow de la Universidad de Stanford (Estados Unidos), en una Conferencia pronunciada durante la Segunda Conferencia Europea sobre Investigación en Aprendizaje e Instrucción, celebrada en Tübingen (República Federal de Alemania), del 19 al 22 de septiembre de 1987, el número de psicólogos existentes en España, más de 300 por millón de habitantes, es semejante al de Estados Unidos, Israel, Suecia, Finlandia y Bélgica. 


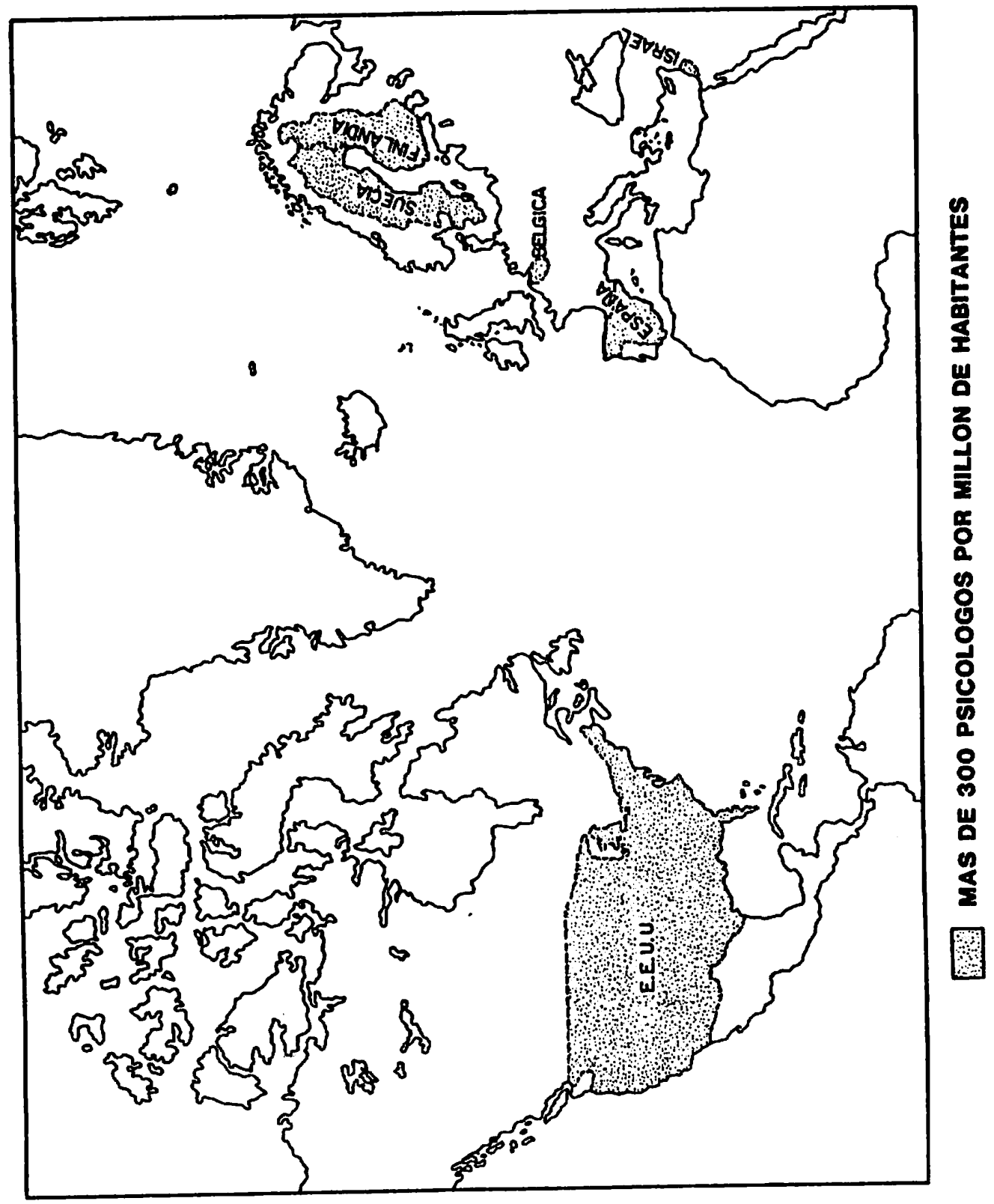

Figura 2 
Creemos que este enorme atractivo que la Psicología ejerce en la actualidad entre los jóvenes se debe al tipo de trabajo que el psicólogo realiza. Este profesional intenta mejorar con su trabajo las condiciones de vida y salud mental de sus conciudadanos. Pero no hay que olvidar que la función del psicólogo, aunque trabaje en campos aplicados, está impreganada de contenido científico, no en vano, la Psicología aplicada se nutre de los conocimientos científicos que la Psicología Básica le aporta.

\section{Investigación Psicọlógica Básica}

Toda la actividad desarrollada en Psicología se centra en dos aspectos fundamentales:

1. La investigasción básica y aplicada que es la que proporciona y desarrolla el conocimiento científico.

2. La utilización de este conocimiento para proporcionar servicios profesionales en los diversos campos aplicados de la Psicología: educativo, clínico y organizacional.

En primer lugar nos referiremos a la investigación básica. En este sentido tenemos que señalar que ésta busca ante todo la verdad científica a través del paradigma propio de investigación que es el paradigma experimental. Paradigma se entiende en este contexto como conjunto de creencias y preconcepciones compartidas por una comunidad científica en un momento determinado. El paradigma experimental se basa en los siguientes criterios o preconcepciones:

1. El experimento se deriva de una teoría psicológica en la que algunos conceptos están ligados a variables.

2. El experimento pone a prueba una o varias hipótesis que se derivan lógicamente de las muestras de sujetos, situaciones experimentales y medidas de las variables dependientes $\mathrm{e}$ independientes utilizadas.

3. El experimento controla adecuadamente las variables que pudieran interactuar con las variables objeto de estudio.

4. Los resultados del experimento son generalizables a una amplia variedad de situaciones.

5. Sus resultados pueden ser aplicados para solucionar importantes problemas humanos fuera del laboratorio.

Cuando se revisan críticamente los logros de la investigación básica en Psicología teniendo en cuenta los criterios propios del paradigma experimental que acabamos de exponer, tenemos que reconocer que los resultados son un poco frustantes, como han señalado Fishman y Neigher (1982). Sin embargo, ésto es comprensible cuando se tiene en cuenta la estructura de los premios o reforzadores que se utilizan en este tereno.

¿Cuáles son estos premios o reforzadores? Revisemos brevemente cuáles y siguen siendo estos premios. El primer lugar lo ocupa la publicación. Todo investigador espera y desea que sus trabajos sean publidados lo antes posible. En segundo lugar, el investigador busca el reconocimiento de sus colegas. El tercer tipo de reforzadores al trabajo del investigador lo constituye la consecución de ayudas de investigación. Quizás el premio o reforzador más importante que un investigador puede recibir es la 
promoción académica que generalmente se debe en último término a las publicaciones que haya realizado.

Todas estas gratificaciones dependen muy estrechamente en último término de la publicación de los trabajos de investigación. Conseguir que los trabajos de un científico sean publicados y conseguir la seguridad en el empleo, ya sea con el sistema americano de la "tenure" o el sistema de funcionariado que tenemos en nuestro país, constituyen las dos principales preocupaciones del docente que empieza su carrera académica. No cabe duda que la publicación ha jugado un papel decisivo en la evolución de la ciencia contemporánea. Como ha señalado Mahoney (1985), desde que en el siglo XVII apareció la primera revista científica a las más de 40.000 revistas científicas actuales, el cambio cuantitativo experimentado ha sido increible. Cada 30 segundos aparece un nuevo artículo científico lo que hace que sólo una parte relativamente pequeña de la literatura pueda ser leida por la comunidad científica.

La mayor preocupación del científico en la actualidad suele ser conseguir que los trabajos que escriba sean publicados porque aquellos científicos que logran publicar su trabajo ven aumentadas las posibilidades de obtener empleo, becas y un trabajo estable, además de poder lograr el reconocimiento por parte de sus colegas. Pero ¿qué pasa con los criterios de publicación? Los criterios actuales de publicación no favorecen precisamente los criterios propios del paradigma experimental y ésto contribuye a que los logros de la investigación básica en Psicología sean limitados.

Veamos brevemente cuáles son los problemas que plantean los criterios de publicación y los que infiuyen en la probabilidad de publicación:

1. En primer lugar, se aprecian decisiones de publicación sesgadas y poco fiables como han demostrado Peters y Ceci (1980). El tema del sesgo en la publicación ha sido también puesto sobre el tapete por la revista The Behavioral and Brain Sciences, volumen 5 junio de 1982. El nombre del autor, así como el prestigio de la institución en la que éste trabaja influyen enormemente en las posibilidades de publicar los trabajos de investigación. Autores eminentes que trabajan en instituciones prestigiosas tienen más facilidad para la publicación de sus trabajos, raramente se les pide que revisen sus trabajos o se les pide que realicen revisiones menores, y cuando ésto ocurre, reciben las revisiones más rápidamente. Se juzga muchas veces por el nombre y no por el contenido de los trabajos, aunque por otro lado, es lógico esperar que un científico eminente produzca un buen trabajo.

2. Se suelen rechazar los trabajos que sena replicación de otro trabajo previo, así como aquellos trabajos que apoyan la hipótesis nula no logrando confirmar la hipótesis o hipótesis propuestas.

3. Como consecuencia de lo anterior, se infla el número de evidencias confirmatorias.

4. Se valora la metodología sofisticada sobre el contenido de la investigación.

5. Se valora poco la relevancia ecológica. En este sentido algunos psicólogos se han quejado de esta falta de relevancia ecológica de las investigaciones. Un psicólogo bien conocido como es Neisser (1976) ha pedido a los psicólogos que reemplacen los flaxes taquistoscópicos y las sílabas sin sentido por la realización de tareas significativas.

Los cuatro primeros criterios tienen mucha importancia para la investigación básica ya que los resultados de investigación tienen un valor limitado, a menos que puedan ser 
replicados y comunicados a través de publicaciones profesionales cditadas de manera fiable y no sesgada. El último criterio es de suma importancia para la Psicología Aplicada.

A modo de resumen podemos señalar que parte de los problemas que la Psicología Básica tiene planteados en la actualidad están relacionados con los incentivos o gratificaciones que se conceden a los profesionales que se dedican a la investigación y, en especial, con la publicación y los sesgos que en su seno se producen.

\section{Investigación aplicada} área:

A nivel de la investigación aplicada existen dos posiciones diferentes dentro de este

1. La que defiende que la investigación aplicada consiste en la aplicación de los principios teóricos derivados de la investigación básica a la solución de importantes problemas psicológicos y sociales.

2. La que defiende que investigación básica e investigación aplicada funcionan con paradigmas diferentes, el de la ciencia y el de la tecnología.

Para los defensores de la primera postura, ésto es, que la investigación aplicada es una extensión de la básica, los problemas que existen en la investigación básica, existen también en la aplicada. Para los que defienden la segunda postura, los logros de la investigación aplicada entendida como tecnologia, han de juzgarse por sus propios méritos. Por tecnología se entiende un procedimiento o secuencia de actividades que conducen a un fin práctico.

\section{Diferencias entre Ciencia y Tecnología}

Ciencia (Invest. Básica)

1. Visión Cartesiana de la Investigación.

2. Tradición de libertad académica.

3. Enfasis en los estudios controlados de laboratorio.

4. Enfasis en la prueba de hipótesis.

5. Enfasis en lo teórico, Conocimiento basado en la teoría.
Tecnología (Invest. Aplicada)

1. Visión Baconiana de la Inv.

2. El área de estudio determinado por las necesidades sociales.

3. Enfasis en la investigación de campo realizada en el mundo real.

4. Enfasis en la observación sistemática.

5. Enfasis en la aplicación práctica. Reducción de los problemas psicológicos.

En el Cuadro 1 pueden verse las diferencias entre investigación básica y aplicada entendidas como dos paradigmas diferentes, el de la ciencia y el de la tecnología. El 
primero está relacionado con la visión Cartesiana de la ciencia y el segundo con la visión Baconiana.

Las diferencias entre ambas posturas pueden resumirse de la siguiente manera:

1. El investigador que adopta la postura Cartesiana y se adapta al paradigma de la ciencia, ve a sus colegas como competidores y lo que le interesa es llegar el primcro a la meta, lograr el primero un determinado descubrimiento. Por el contrario, la posición Baconiana está asociada con el paradigma de la tecnología. La investigación es una actividad cooperativa, de equipo, marcada por una clara división del trabajo y encaminada al logro del bienestar humano.

2. A nivel de investigación, la visión Cartesiana de la ciencia defiende la libcrtad académica. El investigador tiene derccho a investigar lo que le interese. El propio investigador puede decidir su campo de estudio. La visión Baconiana, sin embargo, hace hincapié en que el investigador aplicado debe investigar aquello que un programa claramente articulado de intereses públicos, considere como un problema al que hay que dar una solución.

3. El investigador básico realiza su función normalmente en el seno del laboratorio, en una situación experimental que puede ser perfectamente controlada. $\mathrm{La}$ investigación aplicada se centra en la significación clínica frente a la simplicidad de la respuesta y pone énfasis en la complejidad situacional frente a la simplicidad de los hechos que se estudian en el laboratorio. También le interesan las diferencias individuales y destaca la heterogeneidad de la población frente a la homogeneidad de los sujetos por la que se interesa la ciencia que busca fundamentalmente leyes generales en lugar de excepciones a la regla.

4. La investigación básica desde el paradigma cartesiano pone a prueba hipótesis derivadas de una teoría y la mejor forma de lograr este objetivo es crear situaciones controladas, ésto es, situaciones artificiales creadas en el ámbito del laboratorio. Por otro lado, la tecnología se enfrenta al mundo real. Es necesario que tenga una visión clara y sistemática de los fenómenos sociales. De aquí que use medidas tipificadas y normas a gran escala.

5. El objetivo final de la ciencia es el desarrollo de la teoría mientras que el de la tecnología va encaminado hacia la acción práctica. Podemos decir que el conocimiento basado en teorías equivale a desarrollar un producto de alta calidad y fiabilidad, sin tener en cuenta otros factores, mientras que la preocupación por conseguir una guía de acción práctica equivale a preocuarse no solo de la calidad y fiabilidad del producto, sino también a su facilidad de comercialización, su posibilidad de producción a gran escala, su costo efectivo, etc.

El modelo de la investigación aplicada como tecnología no tiene hoy demasiada aceptación, si se exceptúan ciertos tratamientos de modificación de conducta o la construcción de cuestionarios y tests psicológicos. La mayor parte de las tecnologías desarrolladas por los psicólogos han sido de tipo escrito como son los tests de evaluación psocológica o los entrenamiento separar realizar diversos procedimientos terapéuticos en psicología clínica. Sin embargo, los psicólogos utilizan equipos cada vez más sofisticados equipamientos para desarrollar su actividad, como es el caso de la utilización de sensores eléctricos en procedimientos de biofeedback, y el uso del ordenador para la corrección e interpretación de tests y cuestionarios psicológicos. 
En la actualidad, como ha señalado Crawford (1985), la tecnologia se desarrolla por un equipo compuesto por personas de diferente formación y experiencia que trabajan como grupo. Sus esfucrzos están encaminados a producir una contribución única que ayuda a la resolución del problema que se tiene entre manos, siempre dentro de una visión Baconiana de la tecnología.

La Psicología Aplicada, a través del servicio profesional que dispensa, se implica directamente en los asuntos de cada día. En este punto, la relación que existe entre el estado de la tecnología y la calidad del servicio que proporciona el profesional es muy directa. Cuando un cliente, paciente u organización plantea sus problemas a un profesional, éste no puéde investigar el problema para desarrollar un procedimiento eficaz que lo resuelva. Antes bien, esa persona es un profesional porque tiene a su disposición una serie de procedimientos técnicos y es capaz de seleccionar de entre ellos, aquel que resulta más efectivo para la solución del problema que tiene planteado.

\section{Un plan de acción para la Psicología}

El objetivo más importante de la Psicología en estos momentos es doble. Por un lado, mejorar la calidad de la investigación básica y aplicada. Por otro, unir estos dos tipos de investigación de la manera más estrecha posible con los servicios que la Psicología presta en el ámbito profesional, entrenamiento y formación de nuevos psicólogos, etc.

Como los recursos existentes son escasos, lo más importante que la Psicología tiene que hacer en estos momentos es planificar el uso de estos recursos de una manera explícita y sistemática. Para ello se propone el siguiente plan de acción:

Por un lado, se hace necesaria la reforma de la investigación básica. Como y hemos señalado, los posibles logros de la Psicología están siendo minados por un sistema de reforzadores que no tiene en cuenta los preceptos fundamentales del área. Como posible solución al principal problema que es el de la publicación parece que sería necesario que las revistas españolas y extranjeras exigieran un mínimo de replicaciones de un experimento antes de dar como válido un resultado y aconsejar su publicación. Recordemos, que lo que sucede es justamente lo contrario, las revistas no suelen publicar trabajos que supongan la replicación de trabajos previos. De esta manera, aunque se reduzca el número de publicaciones aumentará el valor de las mismas.

Otro aspecto importante que ayudaría a aumentar el valor práctico de la investigación sería tener en cuenta ciertas normas de importancia ecológica, tales como favorecer la publicación de ciertos artículos que contengan consideraciones explícitas sobre la importancia práctica del experimento y las posibles aplicaciones de los resultados del mismo para la posible solución de problemas psicológicos. Esto no quiere decir que no sean necesarias cierto número de publicaciones dedicadas a temas puramente teóricos o metodológicos, sino que se tenga en cuenta la necesidad de publicar trabajos de relevancia ecológica.

Con respecto al sesgo que se aprecia en la publicación y los peligros que entraña la evaluación por los iguales, parece necesario establecer alguna forma standarizada de evaluación que se debería aplicar a todos los trabajos que llegan a las revistas especializadas para su publicación. 
Por lo que respecta a la investigación aplicada sería de interés que pudieran ponerse en prática algunas de las sugerencias que se hacen a continuación:

- Sería de gran interés que se desarrollara el paradigma de investigación tecnológica y se le diera la misma importancia que al enfoque científico.

- Además, en una sociedad como la nuestra, en la que los recursos dedicados a la investigación y a los programas sociales son muy limitados, éstos deberían dirigirse hacia la solución de problemas sociales. En el caso de que existan varias alternativas o varios programas de intervención diferentes, deberían ser probados y evaluados de manera sistemática con el fin de determinar cual es el que resulta más efectivo y menos costoso.

- También sería bueno, aplicar los conocimientos derivados del campo de la evaluación psicológica a la forma de evaluar a los estudiantes de Psicología de nuestras Universidades. Muchos de los exámenes que se realizan a nuestros estudiantes, se basan demasiado en la superación de pruebas tipo test, de dos alternativas o de alternativas múltiples que en ocasiones carecen de validez como medida de aptitud de nuestros estudiantes sobre su capacidad para prestar servicios profesionales competentes. Sobre todo, durante los últimos cursos se debería medir su capacitación a través de algún procedimiento basado en la actuación.

-Un hecho importante que favorecería la formación de nuestros alumnos consiste en la creación de centros de "servicios integrados". Este es un problema importante, no sólo en nuestro país en el que la Psicología es una especialidad muy nueva, sino también en otros muchos países e los que la Psicología tiene larga tradición, el mundo académico es un mundo separado del mundo en el que se va a desarrollar el trabajo del futuro psicólogo aplicado. Cuando termine su carrera, la mayor parte de los alumnos se dedicará al campo educativo, al campo de la salud pública o al campo de la empresa. El mundo de la formación y el mundo de la profesión continúan siendo dos mundos separados. Una posible solución, aunque no una solución sencila a este problema, podría ser dotar a nuestras facultades de Psicolgía de un "centro de servicios integrados" en el que entren en contacto las aportaciones del mundo académico con la práctica aplicada a la que hace referencia el servicio público al que está abocado nuestra profesión.

Estas son sólo algunas medidas que podrían mejorar tanto el panorama de la psicología básica como aplicada. No pensamos, sin embargo, que sean fáciles de aplicar aunque si creemos que éste puede ser el camino por el que debe dirigirse la Psicología de finales del siglo XX. 


\section{REFERENCIAS BIBLIOGRAFICAS}

BENJAMIN, T. Jr. (1986). - "Why they don't understand us. A history of psychology's public image". American Psychgologist, 41, 941-946.

CRAWFORD, M. (1985). - "Psychology, technology, and professional service". American Psychologist, 40 (4), 415-422.

FISHMAN, D. B. \& NEIGHER, W. D. (1982). - "American psychology in the eighties, who will buy". American Psychologist, 37 (5), 533-546.

MAHONEY, M. J. (1985). - "Open exchange and epistemic progress". American Psychologist, 40 (1), 29-39.

McKINNEY, F. (1976). - "Fifty years of Psychology". American Psychologist, december, 834 842.

PETERS, D. P. \& CECI, S. J. (1982). - "Peer-review practices of psychological journals: The fate of published articles, submitted again". The Behavioral and Brain Sciences, 5, 187 195.

SNOW, R. (1987). - European Research on Learning and Instruction from an American Perspective. Second European Conference on Learning and Instruction. Tubingen, R. F. of Germany, september 19-22, 1987.

THE BEHAVIORAL AND BRAIN SCIENCES, (1982), 5, (2), 185-255. 